\title{
Interpolation of an analytic family of operators on variable exponent Morrey spaces
}

\author{
Alexander Meskhi, Humberto Rafeiro and Muhammad Asad Zaighum \\ (Received April 4, 2017) \\ (Revised February 28, 2018)
}

\begin{abstract}
In this paper we show the validity of Stein's interpolation theorem on variable exponent Morrey spaces.
\end{abstract}

\section{Introduction}

The Stein interpolation theorem, where the interpolation is given with regards to an analytic family of operators, is an essential tool pervading modern Fourier analysis. For example, the first non-trivial progress on spherical summation of multiple Fourier series was obtained with the usage of this theorem, see [7] for more details. Stein's interpolation theorem is given in the framework of Lebesgue spaces and we were not able to find such an interpolation theorem for Morrey spaces. It is interesting to note that the Riesz-Thorin interpolation theorem when the domain space is a Morrey type space does not hold for appropriate counter examples see [18]. Hence, the proved Stein type result will deal only when the target space are Morrey type spaces but the domain is a Lebesgue type space. For interpolation type results on Morrey-Campanato spaces, we refer to $[9,17,28]$ and references therein.

In 1938 C. Morrey [19] studied Morrey spaces for the first time in connection to its applications in partial differential equations. Until recently, a rapid growth has been seen in the study of Morrey type spaces because of its applications in major fields of engineering and sciences (see e.g. [8]). For a comprehensive study of Morrey spaces we refer to [2, 22, 21]. Function spaces with non-standard growth has seen a major focus in recent times (see e.g. $[14,15])$ because of its wide range of applications e.g. in the area of image processing [1, 27], the study of thermorheological fluids [4] and modeling of electrorheological fluids [23].

2010 Mathematics Subject Classification. Primary 46E30, 46B70.

Key words and phrases. Complex interpolation, Stein theorem, variable exponent spaces, Morrey spaces. 
Let $X$ and $Y$ be two quasi-metric measure spaces (QMMSs). In this manuscript, a version of Stein's interpolation theorem is proved in the framework when the target space is a variable exponent Morrey space $L^{q(\cdot), \lambda(\cdot)}(Y)$ and the domain space is the variable exponent Lebesgue space $L^{p(\cdot)}(X)$. It is worth mentioning that these results are new even for the constant case.

Throughout the paper, constants (often different constants in the same series of inequalities) will mainly be denoted by $c$ or $C$; by the symbol $p^{\prime}(x)$ we denote the function $\frac{p(x)}{p(x)-1}, 1<p(x)<\infty$; the relation $a \approx b$ means that there are positive constants $c_{1}$ and $c_{2}$ such that $c_{1} a \leq b \leq c_{2} a$.

\section{Preliminaries}

Let $X$ be a non-empty set. A function $d: X \times X \rightarrow[0, \infty)$ is said to be quasi-metric if the following conditions are satisfied:

(a) $d(x, y)=0$ for all $x \in X$.

(b) $d(x, y)>0$ for all $x, y \in X$ and $x \neq y$.

(c) There is a constant $c_{0}>0$ such that $d(x, y)=c_{0} d(y, x)$ for all $x, y \in X$.

(d) There is a constant $c_{1}>0$ such that $d(x, y) \leq c_{1}(d(x, z)+d(z, y))$ for all $x, y, z \in X$.

Let $\mu$ be a complete measure such that the set of all compactly supported continuous functions are dense in $L_{\mu}^{1}(X)$. We refer the triplet $(X, d, \mu)$ as quasi-metric measure spaces (QMMS), where $d$ is a quasi-metric.

Let $d_{X}=\operatorname{diam}(X)=\sup \{d(x, y): x, y \in X\}$. Let us denote by $B(x, r)=$ $\{y \in X: d(x, y)<r\}$ a ball of radius $r>0$ and centered at $x$. Throughout this paper, it will be assumed that $0<\mu(B(x, r))<\infty$ for every $r>0$ and $x \in X$. It is evident that the assumption that all balls have finite measure together with the condition $d_{X}<\infty$ imply $\mu(X)<\infty$.

Variable exponent spaces. Let $\Omega$ be a $\mu$-measurable set in $(X, \mu)$ with positive measure. We denote:

$$
p^{-}(\Omega):=\inf _{\Omega} p, \quad p^{+}(\Omega):=\sup _{\Omega} p
$$

for a $\mu$-measurable function $p$ on $\Omega$. Suppose that $1 \leq p^{-}(\Omega) \leq p^{+}(\Omega)<\infty$. We say that a $\mu$-measurable function $f$ on $\Omega$ belongs to $L^{p(\cdot)}(\Omega)$ (or to $\left.L^{p(x)}(\Omega)\right)$ if

$$
S_{p(\cdot), \Omega}(f)=\int_{\Omega}|f(x)|^{p(x)} \mathrm{d} \mu(x)<\infty .
$$


It is a Banach space with respect to the norm (see e.g. $[11,16,24,25]$ )

$$
\|f\|_{L^{p(\cdot)}(\Omega)}=\inf \left\{\eta>0: S_{p(\cdot), \Omega}\left(\frac{f}{\eta}\right) \leq 1\right\} .
$$

For the following propositions we refer to $[16,24,25]$.

Proposition 1 (Hölder's inequality). Let $\Omega$ be a $\mu$-measurable subset of $X$ and let $1 \leq p^{-}(\Omega) \leq p^{+}(\Omega)<\infty$. Then for every $f \in L^{p(\cdot)}(\Omega)$ and $g \in L^{p^{\prime}(\cdot)}(\Omega)$ the following inequality

$$
\left|\int_{\Omega} f(x) g(x) \mathrm{d} \mu(x)\right| \leq\left(\frac{1}{p^{-}(\Omega)}+\frac{1}{\left(p^{+}(\Omega)\right)^{\prime}}\right)\|f\|_{L^{p(\cdot)}(\Omega)}\|g\|_{L^{p^{\prime}(\cdot)(\Omega)}}
$$

holds.

The following lemma has been taken from [5, p. 27].

Lemma 1. Let $\Omega$ be a $\mu$-measurable subset of $X$ and let $1 \leq p^{-}(\Omega) \leq$ $p^{+}(\Omega)<\infty$. Then the following inequality

$$
\|f\|_{L^{p(\cdot)}(\Omega)} \leq S_{p(\cdot), \Omega}(f)+1
$$

holds.

Definition 1. We say that a $\mu$-measurable function $p: X \rightarrow[1, \infty)$ belongs to the class $\mathscr{P}_{\mu}^{\log }(X)$ if for every $x, y \in X$ such that $\mu B(x, d(x, y)) \leq$ $1 / 2$ the following inequality

$$
|p(x)-p(y)| \leq \frac{-A}{\ln \mu(B(x, d(x, y)))}
$$

holds.

The following lemma can be found in $[22,14]$.

Lemma 2. Let $(X, d, \mu)$ be a $Q M M S$ with $\mu(X)<+\infty$ and let $p \in \mathscr{P}_{\mu}^{\log }(X)$. Then

$$
\left\|\chi_{B(x, r)}\right\|_{L^{p(\cdot)}} \leq C(\mu(B(x, r)))^{1 / p(x)} .
$$

Morrey spaces with variable exponent $L^{p(\cdot), \lambda(\cdot)}(\Omega)$ where $\Omega$ is an open subset of $\mathbb{R}^{n}$ were introduced simultaneously by Almeida et al. [3], Kokilashvili et al. $[12,13]$, Ohno [20] and X. Fan [6] in more or less similar manner. Let $1 \leq p(\cdot)<p^{+}(\Omega)<\infty$ and $0 \leq \lambda(\cdot) \leq 1$ be $\mu$-measurable functions. We say that a $\mu$-measurable function $f \in L^{p(\cdot)}(\Omega)$ belongs to $L^{p(\cdot), \lambda(\cdot)}(\Omega)$ if

$$
I_{p(\cdot), \lambda(\cdot)}(f)=\sup _{x \in \Omega, r>0} \frac{1}{(\mu(B(x, r)))^{\lambda(x)}} \int_{B(x, r)}|f(y)|^{p(y)} \mathrm{d} \mu(y)<\infty .
$$


The norm on variable exponent Morrey spaces can be introduced in the following ways (see e.g. $[3,12,13,22])$ :

$$
\|f\|_{1}=\inf \left\{\eta>0: I_{p(\cdot), \lambda(\cdot)}(f / \eta) \leq 1\right\}
$$

and

$$
\|f\|_{2}=\sup _{x \in \Omega, r>0}\|(\mu(B(x, r)))\|^{-\lambda(x) / p(\cdot)} f \chi_{B(x, r) L^{p(\cdot)}(\Omega)},
$$

and

$$
\|f\|_{3}=\sup _{x \in \Omega, r>0}(\mu(B(x, r)))^{-\lambda(x) / p(x)}\|f\|_{L^{p(\cdot)}(B(x, r))} .
$$

It can be checked easily by means of simple computations that $\|f\|_{1}=\|f\|_{2}$. Further, if the exponent $p$ is such that $p \in \mathscr{P}_{\mu}^{\log }(X)$ (see e.g. [22]) then both the norms $\|f\|_{2}$ and $\|f\|_{1}$ are equivalent to $\|f\|_{3}$. We define the norm on variable exponent Morrey space as:

$$
\|f\|_{L^{p(\cdot), \lambda(\cdot)}(X)}=\|f\|_{3} .
$$

It is easy to see that if the parameter $\lambda=0$, then $L^{p(\cdot)}(X)=L^{p(\cdot), 0}(X)$. When $p(x) \equiv$ const and $\lambda(x) \equiv$ const then $L^{p(\cdot), \lambda(\cdot)}(X)$ is reduced to the case of classical Morrey space $L^{p, \lambda}(X)$.

The following lemma gives the embedding of variable Morrey spaces into variable Lebesgue space in the case $d_{X}<\infty$. Here we present the proof of this lemma for the sake of completeness.

Lemma 3. Let $(X, d, \mu)$ be a $Q M M S$. Suppose that $1 \leq p(\cdot)<p^{+}(X)$ $<\infty$ and $0 \leq \lambda(\cdot) \leq 1$. Then for every $f \in L^{p(\cdot), \lambda(\cdot)}(X), x \in X$ and $r>0$ we have

$$
\|f\|_{L^{p(\cdot)}(B(x, r))} \leq(\mu(B(x, r)))^{\lambda(x) / p(x)}\|f\|_{L^{p(\cdot), \lambda(\cdot)}(X)} .
$$

Moreover, if $\mu(X)<\infty$ then

$$
\|f\|_{L^{p(\cdot)}(X)} \leq c_{p, \lambda, \mu}\|f\|_{L^{p(\cdot), \lambda(\cdot)}(X)} .
$$

Proof. Suppose that $f \in L^{p(\cdot), \lambda(\cdot)}(X)$. Let $x \in X$ and $r>0$, then

$$
\begin{aligned}
\|f\|_{L^{p(\cdot)}(B(x, r))} & =(\mu(B(x, r)))^{\lambda(x) / p(x)} \frac{1}{(\mu(B(x, r)))^{\lambda(x) / p(x)}}\|f\|_{L^{p(\cdot)}(B(x, r))} \\
& \leq(\mu(B(x, r)))^{\lambda(x) / p(x)}\|f\|_{L^{p(\cdot), \lambda(\cdot)}(X)} .
\end{aligned}
$$

Since $p$ is bounded, hence taking supremum with respect to $x \in X$ and $r>0$ we have the following estimate 


$$
\begin{aligned}
\|f\|_{L^{p(\cdot)}(X)} & \leq \max \left\{1,(\mu(X))^{(\lambda / p)^{+}(X)}\right\}\|f\|_{L^{p(\cdot), \lambda(\cdot)}(X)} \\
& \leq c_{p, \lambda, \mu}\|f\|_{L^{p(\cdot), \lambda(\cdot)}(X)} .
\end{aligned}
$$

Consequently, via Hölder's inequality, for $f \in L^{p(\cdot), \lambda(\cdot)}(X)$ and $g \in L^{p^{\prime}(\cdot)}(X)$ there is a positive constant $c$ such that,

$$
\int_{X} f(y) g(y) \mathrm{d} \mu(y) \leq c\|f\|_{L^{p(\cdot), \lambda(\cdot)}(X)}\|g\|_{L^{p^{\prime}(\cdot)}(X)}
$$

holds.

\section{Interpolation of analytic family of operators in variable exponent Morrey spaces}

In this section we prove the main result of this paper. We prove the Stein interpolation type theorem for analytic family of operators.

Definition 2. A function $f(z)$ analytic on an open strip $0<\operatorname{Re}(z)<1$ and continuous and bounded on the closed strip is said to be of admissible growth if for $a<\pi$ the following inequality

$$
\sup _{|y| \leq r} \sup _{0 \leq x \leq 1}|f(x+\mathrm{i} y)| \leq C \mathrm{e}^{a r},
$$

holds, where $C$ is a positive constant.

The next lemma is due to Hirschman and can be found in e.g. [10].

Lemma 4 (Hirschman Lemma). Let $f(z)$ be analytic on an open strip $0<\operatorname{Re}(z)<1$ and continuous and bounded on the closed strip and of admissible growth there. Let

$$
\log |f(\mathrm{i} y)| \leq A_{0}(y), \quad \log |f(1+\mathrm{i} y)| \leq A_{1}(y),
$$

then for $0 \leq t \leq 1$ the following inequality

$$
\begin{aligned}
\log |f(t)| \leq( & \frac{1}{2} \int_{-\infty}^{\infty} \frac{\sin (\pi t)}{\cosh (\pi y)-\cos (\pi t)} A_{0}(y) \mathrm{d} y \\
& \left.+\frac{1}{2} \int_{-\infty}^{\infty} \frac{\sin (\pi t)}{\cosh (\pi y)+\cos (\pi t)} A_{1}(y) \mathrm{d} y\right)
\end{aligned}
$$

holds.

Definition 3 (Analytic Family of Operators). Let $\left(X_{1}, d_{1}, \mu_{1}\right)$ and $\left(X_{2}, d_{2}, \mu_{2}\right)$ be QMMSs. Consider a family of linear operators $\left\{T_{z}\right\}_{z \in \mathbb{C}}$. We shall call this family of linear operators to be analytic if: 
(1) For each $z \in \mathbb{C}, T_{z}$ maps simple functions in $\left(X_{1}, d_{1}, \mu_{1}\right)$ on measurable functions in $\left(X_{2}, d_{2}, \mu_{2}\right)$.

(2) For $z \in \mathbb{S}, r>0$ and a.e. $y \in X_{2}$, the function $F_{y, r}(z)$ defined by

$$
\begin{aligned}
F_{y, r}(z):= & \int_{B(y, r)} T_{z}\left[a_{1}^{m_{1}(\cdot) z+b_{1}(\cdot)} \chi_{A_{1}}(\cdot)\right]\left(x_{2}\right) \\
& \times a_{2}^{m_{2}\left(x_{2}\right) z+b_{2}\left(x_{2}\right)} \chi_{A_{2}}\left(x_{2}\right) \mathrm{d} \mu_{2}\left(x_{2}\right),
\end{aligned}
$$

exists, is continuous and bounded on the strip $\mathbb{S}=\{z: 0 \leq \operatorname{Re}(z) \leq$ $1\}$ and analytic on $\operatorname{int}(\mathbb{S})$, where $a_{k}$ are positive real numbers and $m_{k}$, $b_{k}$ are measurable functions for $k=1,2$.

We shall call $\left\{T_{z}\right\}_{z \in \mathbb{C}}$ of admissible growth if $F_{y, r}(z)$ is of admissible growth in the sense of Definition 2.

REMARK 1. Although the definition of an analytic family of operators given in Definition 3 seems cumbersome at first sight, but it should be noted that in the non-variable framework this definition coincides with the definition given by Stein in [26].

We now formulate and prove the Stein interpolation theorem in the variable exponent framework.

THEOREM 1. Let $(X, \mu)$ and $(Y, v)$ be $\sigma$-finite, complete QMMSs. For $k=0,1$, assume that $1 \leq p_{k}(\cdot), q_{k}(\cdot)<q_{k}^{+}(Y)<\infty$ and $0 \leq \lambda_{k}(\cdot) \leq 1$. Suppose that we have an analytic family of linear operators $T_{z}: L^{p_{k}(\cdot)}(X) \rightarrow L^{q_{k}(\cdot), \lambda_{k}(\cdot)}(Y)$ which is of admissible growth in the strip $\overline{\mathbb{S}}:=\{z: 0 \leq \operatorname{Re}(z) \leq 1\}$. Further suppose that the following inequalities

$$
\begin{gathered}
\left\|T_{\mathrm{i} t} f\right\|_{L^{q_{0}(\cdot), \lambda_{0}(\cdot)}(Y)} \leq M_{0}(t)\|f\|_{L^{p_{0}(\cdot)}(X)} \\
\left\|T_{1+\mathrm{i} t} f\right\|_{L^{q_{1}(\cdot), \lambda_{1}(\cdot)(Y)}} \leq M_{1}(t)\|f\|_{L^{p_{1}(\cdot)}(X)}
\end{gathered}
$$

hold for all simple functions $f$. Also we assume that

$$
\log \left|M_{k}(t)\right| \leq C \mathrm{e}^{|t| l} \quad l<\pi \text { for } k=0,1 .
$$

For $z \in \mathbb{S}:=\{z: 0<\operatorname{Re}(z)<1\}$, define $p_{z}, q_{z}$ and $\lambda_{z}$ by

$$
\begin{aligned}
& \frac{1}{p_{z}(x)}=\frac{1-z}{p_{0}(x)}+\frac{z}{p_{1}(x)}, \\
& \frac{1}{q_{z}(x)}=\frac{1-z}{q_{0}(x)}+\frac{z}{q_{1}(x)},
\end{aligned}
$$

and

$$
\frac{\lambda_{z}(x)}{q_{z}(x)}=(1-z) \frac{\lambda_{0}(x)}{q_{0}(x)}+z \frac{\lambda_{1}(x)}{q_{1}(x)} .
$$


Then, given any $\theta \in(0,1)$, the inequality

$$
\left\|T_{\theta} f\right\|_{L^{q_{\theta}^{(\cdot), \lambda_{\theta}(\cdot)}(Y)}} \leq c M_{\theta}\|f\|_{L^{p_{\theta}^{(\cdot)}(X)}}
$$

holds for every $f \in L^{p_{\theta}(\cdot)}(X)$, where

$$
\begin{aligned}
\log M_{\theta}= & \left(\frac{1}{2} \int_{-\infty}^{\infty} \frac{\sin (\pi \theta)}{\cosh (\pi y)-\cos (\pi \theta)} \log M_{0}(y) \mathrm{d} y\right. \\
& \left.+\frac{1}{2} \int_{-\infty}^{\infty} \frac{\sin (\pi \theta)}{\cosh (\pi y)+\cos (\pi \theta)} \log M_{1}(y) \mathrm{d} y\right) .
\end{aligned}
$$

Proof. Since $T_{z}$ is linear, we may assume that $f \neq 0$, otherwise the inequality holds for $f=0$. By the homogeneity of the norm and the scaling argument we may assume that $\|f\|_{L^{p_{\theta}(\cdot)}(X)} \leq 1$. Now we need to show that

$$
\left\|T_{z} f\right\|_{L^{q_{\theta}(\cdot), \lambda_{\theta}(\cdot)}(Y)} \leq c M_{\theta} \text {. }
$$

We will show (6) for simple functions in $X$ and since the span of simple functions is dense in $L^{p(\cdot)}(X)$ we will have the estimate for all $f \in L^{p_{\theta}(\cdot)}(X)$.

Let us assume $f, g$ are simple and complex valued functions defined on $X$ and $Y$ respectively by,

$$
\begin{array}{ll}
f(x)=\sum_{j=1}^{m} a_{j} \mathrm{e}^{\mathrm{i} \alpha_{j}} \chi_{A_{j}}(x), & x \in X \\
g(y)=\sum_{k=1}^{n} b_{k} \mathrm{e}^{\mathrm{i} \beta_{k}} \chi_{B_{k}}(y), \quad y \in Y
\end{array}
$$

where $a_{j}, b_{k}>0$ and $\alpha_{j}, \beta_{k} \in \mathbb{R}, \mu\left(A_{j}\right), \mu\left(B_{k}\right)<\infty$, and $\left\{A_{j}\right\}$ and $\left\{B_{k}\right\}$ are, respectively, pairwise disjoint. Now define,

$$
\begin{aligned}
& f_{z}(x)=\sum_{j=1}^{m} a_{j}^{p_{\theta}(x) / p_{z}(x)} \mathrm{e}^{\mathrm{i} \alpha_{j}} \chi_{A_{j}}(x), \\
& g_{z}(y)=\sum_{k=1}^{n} b_{k}^{q_{\theta}^{\prime}(y) / q_{z}^{\prime}(y)} \mathrm{e}^{\mathrm{i} \beta_{k}} \chi_{B_{k}}(y) .
\end{aligned}
$$

Finally, for every $y \in Y, r>0$ and $z \in \mathbb{C}$, we put

$$
F_{y, r}(z):=\int_{B(y, r)} T_{z}\left(f_{z}(s)\right) g_{z}(s) \mathrm{d} v(s) .
$$

Substituting the values of $f_{z}$ and $g_{z}$ in the last expression we have

$$
F_{y, r}(z)=\sum_{j=1}^{m} \sum_{k=1}^{n} \int_{B(y, r)} T_{z}\left[a_{j}^{p_{\theta}(\cdot) / p_{z}(\cdot)} \chi_{A_{j}}(\cdot)\right](s) b_{k}^{q_{\theta}^{\prime}(s) / q_{z}^{\prime}(s)} \chi_{B_{k}}(s) \mathrm{d} v(s) .
$$


Hence for almost every $y \in Y, F_{y, r}(z)$ is analytic on $\operatorname{int}(\mathbb{S})$ and continuous and bounded on $\mathbb{S}$ and of admissible growth, since $T_{z}$ is an analytic family of linear operators of admissible growth.

Since $A_{j}$ are pairwise disjoint and $a_{j}>0$, we have for $z=\mathrm{i} t(t \in \mathbb{R})$

$$
\begin{aligned}
S_{p_{0}(\cdot), B(y, r)}\left(f_{z}\right) & =\int_{B(y, r)}\left|\sum_{j=1}^{m} a_{j}^{p_{\theta}(x) / p_{z}(x)} \mathrm{e}^{\mathrm{i} \alpha_{j}} \chi_{A_{j}}(x)\right|^{p_{0}(x)} \mathrm{d} \mu(x) \\
& =\int_{B(y, r)}\left|\sum_{j=1}^{m} a_{j}^{p_{\theta}(x)\left[1 / p_{1}(x)-1 / p_{0}(x)\right] \mathrm{i} t+p_{\theta}(x) / p_{0}(x)} \mathrm{e}^{\mathrm{i} \alpha_{j}} \chi_{A_{j}}(x)\right|^{p_{0}(x)} \mathrm{d} \mu(x) \\
& =\int_{B(y, r)} \sum_{j=1}^{m}\left|a_{j}^{p_{\theta}(x)\left[1 / p_{1}(x)-1 / p_{0}(x)\right] \mathrm{i} t+p_{\theta}(x) / p_{0}(x)} \mathrm{e}^{\mathrm{i} \alpha_{j}} \chi_{A_{j}}(x)\right|^{p_{0}(x)} \mathrm{d} \mu(x) \\
& =\int_{B(y, r)} \sum_{j=1}^{m} a_{j}^{p_{\theta}(x)} \chi_{A_{j}}(x) \mathrm{d} \mu(x) \\
& =\int_{B(y, r)}\left|\sum_{j=1}^{m} a_{j} \mathrm{e}^{\mathrm{i} \alpha_{j}} \chi_{A_{j}}(x)\right|^{p_{\theta}(x)} \mathrm{d} \mu(x) \\
& =S_{p_{\theta}(\cdot), B(y, r)}(f) \\
& \leq 1
\end{aligned}
$$

since $\|f\|_{L^{p_{\theta}(\cdot)}(X)} \leq 1$. Hence $\left\|f_{z}\right\|_{L^{p_{0}(\cdot)}(B(y, r))} \leq 1$. A similar argument shows that $\left\|g_{z}\right\|_{L^{q_{0}^{\prime}(\cdot)}(B(y, r))} \leq 1$ for $z=\mathrm{i} t$. Now by Hölder's inequality, Lemma 3 and (3) we have

$$
\begin{aligned}
\left|F_{y, r}(\mathrm{i} t)\right| & \leq\left|\int_{B(y, r)} T\left(f_{z}(s)\right) g_{z}(s) \mathrm{d} v(s)\right| \\
& \leq c\left\|T f_{z}\right\|_{L^{q_{0}(\cdot)}(B(y, r))}\left\|g_{z}\right\|_{L^{q^{\prime}(\cdot)}(B(y, r))} \\
& \leq c\left\|T f_{z}\right\|_{L^{q_{0}(\cdot)}(B(y, r))} \\
& \leq c(v(B(y, r)))^{\lambda_{0}(y) / q_{0}(y)}\left\|T f_{z}\right\|_{L^{q_{0}(\cdot), \lambda_{0}(\cdot)}(Y)} \\
& \leq c(v(B(y, r)))^{\lambda_{0}(y) / q_{0}(y)} M_{0}(t)\left\|f_{z}\right\|_{L^{p_{0}(\cdot)}(X)} \\
& \leq c(v(B(y, r)))^{\lambda_{0}(y) / q_{0}(y)} M_{0}(t) .
\end{aligned}
$$

An analogous argument with $\operatorname{Re}(z)=1$ for the exponents $p_{1}$ and $q_{1}$ yields,

$$
\left|F_{y, r}(1+\mathrm{i} t)\right| \leq c(v(B(y, r)))^{\lambda_{1}(y) / q_{1}(y)} M_{1}(t) .
$$


Invoking Hirschman's Lemma we have:

$$
\begin{aligned}
\log \left|F_{y, r}(\theta)\right| \leq & \frac{1}{2} \int_{-\infty}^{\infty} \frac{\sin (\pi \theta)}{\cosh (\pi s)-\cos (\pi \theta)} \log \left((v(B(y, r)))^{\lambda_{0}(y) / q_{0}(y)} M_{0}(s)\right) \mathrm{d} s \\
& \left.+\frac{1}{2} \int_{-\infty}^{\infty} \frac{\sin (\pi \theta)}{\cosh (\pi s)+\cos (\pi \theta)} \log \left((v(B(y, r)))^{\lambda_{1}(y) / q_{1}(y)} M_{1}(s)\right) \mathrm{d} s\right) \\
\leq & \frac{\log (v(B(y, r)))^{\lambda_{0}(y) / q_{0}(y)}}{2} \int_{-\infty}^{\infty} \frac{\sin (\pi \theta)}{\cosh (\pi s)-\cos (\pi \theta)} \mathrm{d} s \\
& \left.+\frac{\log (v(B(y, r)))^{\lambda_{1}(y) / q_{1}(y)}}{2} \int_{-\infty}^{\infty} \frac{\sin (\pi \theta)}{\cosh (\pi s)+\cos (\pi \theta)} \mathrm{d} s\right)+\log M_{\theta} .
\end{aligned}
$$

By making the change of variables $\mathrm{e}^{\pi s}=u$ in the above integrals we have

$$
\frac{1}{2} \int_{-\infty}^{\infty} \frac{\sin (\pi \theta)}{\cosh (\pi s)-\cos (\pi \theta)} \mathrm{d} s=1-\theta
$$

and

$$
\frac{1}{2} \int_{-\infty}^{\infty} \frac{\sin (\pi \theta)}{\cosh (\pi s)+\cos (\pi \theta)} \mathrm{d} s=\theta
$$

Hence,

$$
\begin{aligned}
\log \left|F_{y, r}(\theta)\right| \leq & (1-\theta) \log (v(B(y, r)))^{\lambda_{0}(y) / q_{0}(y)} \\
& +\theta \log (v(B(y, r)))^{\lambda_{1}(y) / q_{1}(y)}+\log M_{\theta} \\
\leq & \log (v(B(y, r)))^{(1-\theta)\left(\lambda_{0}(y) / q_{0}(y)\right)}+\log (v(B(y, r)))^{\theta\left(\lambda_{1}(y) / q_{1}(y)\right)}+\log M_{\theta} \\
\leq & \log (v(B(y, r)))^{(1-\theta)\left(\lambda_{0}(y) / q_{0}(y)\right)+\theta\left(\lambda_{1}(y) / q_{1}(y)\right)}+\log M_{\theta} \\
\leq & \log (v(B(y, r)))^{\lambda_{\theta}(y) / q_{\theta}(y)}+\log M_{\theta}
\end{aligned}
$$

which yields

$$
\left|F_{y, r}(\theta)\right| \leq c(v(B(y, r)))^{\lambda_{\theta}(y) / q_{\theta}(y)} M_{\theta}
$$

Also,

$$
\sup _{\|g\|_{L^{q_{\theta(B(y, r))}^{\prime}}} \leq 1} F_{y, r}(\theta) \sim\left\|T_{\theta} f\right\|_{L^{q_{\theta}(\cdot)}(B(y, r))} .
$$

Hence for almost every $y \in Y$ and $r>0$ we have,

$$
(v(B(y, r)))^{-\lambda_{\theta}(y) / q_{\theta}(y)}\left\|T_{\theta} f\right\|_{L^{q_{\theta}^{(\cdot)}(B(y, r))}} \leq c M_{\theta},
$$


which implies that,

$$
\left\|T_{\theta} f\right\|_{L^{q_{\theta}(\cdot), \lambda_{\theta}(\cdot)(Y)}} \leq c M_{\theta} .
$$

This completes the proof.

\section{Acknowledgement}

The second named author was partially supported by research project "Study of boundedness of operators in generalized Morrey spaces", ID-PRJ: 6576 of the Faculty of Sciences of Pontificia Universidad Javeriana, Bogotá, Colombia. The third named author was supported by Pontificia Universidad Javeriana, Bogotá, Colombia as Post-Doctoral Investigator working on the research project "Study of boundedness of some operators in generalized Morrey spaces", ID-PRJ: 6576 (Contract Number: DPE-040-15).

The authors are grateful to the referee for useful remarks and suggestions.

\section{References}

[1] R. Aboulaich, D. Meskine and A. Souissi, New diffusion models in image processing, Comput. Math. Appl., 56 (2008), 874-882.

[2] D. Adam, Morrey Spaces, Lecture Notes in Applied and Numerical Harmonic Analysis, Birkhäuser/Springer, Cham, 2015.

[3] A. Almeida, J. Hasanov and S. Samko, Maximal and potential operators in variable exponent Morrey spaces, Georgian Math. J., 15(2) (2008), 195-208.

[4] S. Antontsev and J. Rodrigues, On stationary thermo-rheological viscous flows, Ann. Univ. Ferrara Sez. VII Sci. Mat., 52 (2006), 19-36.

[5] L. Diening, P. Harjulehto, P. Hästö and M. Ružička, Lebesgue and Sobolev spaces with variable exponents, Lecture Notes in Mathematics, Springer, Heidelberg, 2011.

[6] X. Fan, Variable exponent Morrey and Campanato spaces, Nonlinear Anal., 72(11) (2010), 4148-4161.

[7] C. Fefferman, Selected theorems by Elias Stein in: Advances in analysis. The legacy of Elias M. Stein. Proceedings of the conference "Analysis and applications" held in honor of the 80th birthday of Elias M. Stein, Princeton, NJ, USA, May 16-20, 2011, Princeton, NJ: Princeton University Press, 2014, 1-34.

[8] K. Fey and M. Foss, Morrey regularity for almost minimizers of asymptotically convex functionals with nonstandard growth, Forum Math., 25(5) (2013), 887-929.

[9] D. Hakim and Y. Sawano, Interpolation of generalized Morrey spaces, Rev. Math. Comp., 29(2) (2016), 295-340.

[10] I. Hirschman, A convexity theorem for certain groups of transformations, J. Anal. Math., 2 (1953), 209-218.

[11] V. Kokilashvili, On a progress in the theory of integral operators in weighted Banach function spaces, FSDONA04 Proceedings, Milovy, Czech Republic, 2004.

[12] V. Kokilashvili and A. Meskhi, Boundedness of maximal and singular operators in Morrey spaces with variable exponent, Armen. J. Math., 1(1) (2008), 18-28. 
[13] V. Kokilashvili and A. Meskhi, Maximal functions and potentials in variable exponent Morrey spaces with non-doubling measure, Complex Var. Elliptic Equ., 55 (2010), 923-936.

[14] V. Kokilashvili, A. Meskhi, H. Rafeiro and S. Samko, Integral Operators in Non-Standard Function Spaces. Volume 1: Variable Exponent Lebesgue and Amalgam Spaces, Oper. Theory Adv. Appl. Vol 248, Birkhäuser Verlag, Basel, 2016.

[15] V. Kokilashvili, A. Meskhi, H. Rafeiro and S. Samko, Integral Operators in Non-Standard Function Spaces. Volume 2: Variable Exponent Hölder, MorreyCampanato and Grand Spaces, Oper. Theory Adv. Appl. Vol 249, Birkhäuser Verlag, Basel, 2016.

[16] O. Kováčik and J. Rákosník, On spaces $L^{p(x)}$ and $W^{k, p(x)}$, Czechoslovak Math. J., 116(4) (1991), 592-618.

[17] Y. Lu, D. Yang and W. Yuan, Interpolation of Morrey spaces on metric measure spaces, Canad. Math. Bull., 57(3) (2014), 598-608.

[18] A. Meskhi, H. Rafeiro and M. A. Zaighum, Interpolation on variable Morrey spaces defined on quasi-metric measure spaces., J. Funct. Anal., 270(10) (2016), 3946-3961.

[19] C. Morrey, On the solutions of quasi-linear elliptic partial differential equations, Trans. Amer. Math. Soc., 43(1) (1938), 126-166.

[20] T. Ohno, Continuity properties for logarithmic potentials of functions in Morrey spaces of variable exponent, Hiroshima Math. J., 38(3) (2008), 363-383.

[21] H. Rafeiro, N. Samko and S. Samko, Morrey-Campanato spaces: an overview: Operator theory, pseudo-differential equations, and mathematical physics, Oper. Theory Adv. Appl., Birkhäuser/Springer Basel AG, 2013.

[22] H. Rafeiro and S. Samko, Variable exponent Campanato spaces, J. Math. Sci., 172(1) (2011), 143-164.

[23] M. Ružička, Electrorheological fluids: modeling and mathematical theory, Lecture Notes in Mathematics 1748, Springer-Verlag, Berlin, 2000.

[24] S. Samko, Convolution type operators in $L^{p(x)}$, Integral Transform. Spec. Funct., 1-2 (1998), 123-144.

[25] I. Sarapudinov, The topology of the space $\mathscr{L}^{p(t)}([0,1]), \quad$ Mat. Zametki., 26 (1979), 613-632.

[26] E. Stein, Interpolation of linear operators, Trans. Am. Math. Soc., 83 (1956), 482-492.

[27] T. Wunderli, On time flows of minimizers of general convex functionals of linear growth with variable exponent in BV space and stability of pseudosolutions, J. Math. Anal. Appl., 364(2) (2010), 591-598.

[28] W. Yuan, W. Sickel and D. Yang, Interpolation of Morrey-Campanato and related smoothness spaces., Sci. China, Math., 58(9) (2015), 1835-1908.

\author{
Alexander Meskhi \\ Department of Mathematical Analysis \\ A. Razmadze Mathematical Institute of \\ I. Javakhishvili Tbilisi State University \\ 6. Tamarashvili Str., Tbilisi 0177, Georgia \\ and \\ Department of Mathematics \\ Faculty of Informatics and Control Systems \\ Georgian Technical University \\ 77, Kostava St., Tbilisi, Georgia \\ E-mail:meskhi@rmi.ge
}




\section{Humberto Rafeiro}

Pontificia Universidad Javeriana

Departamento de Matemáticas

Cra. 7, Bogotá, Colombia

E-mail: silva-h@javeriana.edu.co

Muhammad Asad Zaighum

Department of Mathematics and Statistics

Riphah International University

I-14, Islamabad, Pakistan

and

Pontificia Universidad Javeriana

Departamento de Matemáticas

Cra. 7, Bogotá, Colombia

E-mail:asadzaighum@gmail.com 